Memorial Lecture: 1999 JSPM Award for Innovatory Research

\title{
Study on Grain Growth of Fine Grained WC-Co Hardmetal By Numerical Calculation
}

\author{
Naoki Matsuoka ${ }^{\text {मे } 1}$ and Koji Hayashi ${ }^{\text {2 } 2}$ \\ ${ }^{1}$ Kobe Steel, Ltd., 1-5-5 Takatsukadai, Nishi-ku, Kobe 651-2271. \\ Institute of Industrial Science, the University of Tokyo, 7-22-1 Roppongi, Minato-ku, Tokyo 106-8558.
}

Received June 22, 2000

\section{SYNOPSIS}

This paper reviews our simulation study by numerical calculation based on two-, three- and multi-grain-size models on WC grain growth in fine grained WC-Co hardmetal doped with VC. The study aimed to presume or predict the following: (1) the cause and conditions for the abnormal grain growth which occurs in some cases in the fine grained hardmetal prepared from fine WC powders with mean grain size below about $0.2 \mu \mathrm{m}$, and (2) how the mean grain size of the hardmetal varies with decreasing mean grain size of the WC starting powder to $0.1 \mu \mathrm{m}$ or nano-meter size. The calculation results by these three kinds of models suggested the following, respectively: (1) the occurrence of the abnormal grain growth is generally substantial for WC starting powder with bimodal size-distribution and mean grain size below $0.1 \sim 0.2 \mu \mathrm{m}$, and not due to the non-uniform distribution of the grain growth inhibitor, (2) the introduction of middle grains caused the disappearance of small (fine) grains during sintering, leading to abruptly increase of the mean grain size of alloy, and (3) the mean grain size of alloy becomes so large as about $0.3 \sim 0.4 \mu \mathrm{m}$, even when the WC starting powders with nano-size as well as $0.1 \mu \mathrm{m}$ are used.

KEY WORDS

hardmetal, fine grain, WC, grain growth, abnormal grain growth, numerical calculation, simulation

\section{Introduction}

WC-Co base fine grained hardmetals with mean grain size of $0.5 \sim 0.7 \mu \mathrm{m}$ are used as materials of tools such as piercing drills for PCB (printed circuit board), shear blade for paper and magnetic tape, and end mill for cast iron and steel parts, etc. ${ }^{1,2)}$. The hardmetal is a kind of particle dispersion strengthened alloys. This is produced by powder metallurgical process: the liquid-phase sintering of the mixed powder compact which consists of WC of about $0.5 \mu \mathrm{m}$ as hard grains, Co of $1.5 \mu \mathrm{m}$ as metallic binder matrix phase and also a small amount of $\mathrm{VC}, \mathrm{Cr}_{3} \mathrm{C}_{2}$ and/or $\mathrm{TaC}$ of $2 \sim 3 \mu \mathrm{m}$ as WC grain growth inhibitor (the effectiveness is $\mathrm{VC}>\mathrm{Cr}_{3} \mathrm{C}_{2}>\mathrm{TaC}^{3)}$ ). The mechanical properties such as hardness and wear resistance of the alloy are suggested to increase with decreasing mean grain size to less than $0.5 \mu \mathrm{m}$, based on the experimental relation between the mechanical properties and grain size, which was observed in the grain size range above $0.5 \mu \mathrm{m}$. On the other hand, WC fine powders with mean grain size of $0.035 \mu \mathrm{m}$ as well as $0.2 \sim$ $0.3 \mu \mathrm{m}$ have been commercially available from $1996 \sim$ $1997^{4,5)}$.

One reason why the mean grain sizes of commercial fine grained hardmetals are selected above about $0.5 \mu \mathrm{m}$ was suggested to be the avoidance of the abnormal grain growth of WC, taking into consideration a previous experimental result that the abnormal grain growth occurred considerably, when the mean grain size became less than about $0.2 \mu \mathrm{m}$, as shown in Fig. $1^{6}$. Here, the mean grain size was measured by use of Fullman's equation ${ }^{7}$. The causes or conditions of the abnormal grain growth in fine grained WC-Co hardmetal were not clear, although the following hypotheses for the cause were thought; (i) wide WC gain size distribution in the WC starting powder, WC+Co powder compact or initial sintered compact, (ii) non-uniform mixing or distribution of the grain growth inhibitor in the powder compact, and (iii) almost intrinsic phenomenon as far as $\mathrm{VC}, \mathrm{Cr}_{3} \mathrm{C}_{2}$ and/or $\mathrm{TaC}$ are used as the grain growth inhibitor. The well-known equation of the dependence of mean grain size on the heating (sintering) time ${ }^{8.9}$ is not able to resolve which hypothesis among these is the most appropriate: the equation, for example, in the case where the ratedetermining step is the interfacial reaction ${ }^{8,97}$ is the following:

$$
\bar{d}_{t}^{2}-\bar{d}_{0}^{2}=(32 / 81) 4\left(2 \sigma \kappa C_{0} V_{m}^{2} / \mathrm{MR} T\right) t
$$




$$
\bar{d}_{t}^{2}-\bar{d}_{0}^{2}=K t \quad\left\{K \equiv(32 / 81) 4\left(2 \sigma \kappa C_{0} V_{m}^{2} / v \mathrm{R} T\right) t\right\}
$$

where $\bar{d}_{t}$ and $\bar{d}_{0}$ are the mean grain size after sintering time $(t)$ and the initial mean grain size, respectively, $\sigma$ is the interfacial energy between solid and liquid phases, $\kappa$ is the interfacial reaction rate constant, $C_{0}$ is the equilibrium solubility (molar concentration) from bulky solid, $V_{m}$ is the molar volume of solid, $v$ is a correction factor which depends on the deviation of the composition from the stoichiometric value, shape and contiguity of solid grains $^{8,10,11)}, R$ is gas constant and $T$ is the heating (sintering) temperature.

Therefore, clarification of the cause for the abnormal grain growth and also the prediction of how the mean grain size in the hardmetal varies by decreasing the mean grain size of the WC starting powder to below $0.1 \mu \mathrm{m}$ were thought to be important for the determination of the strategy for the future development of fine grained hardmetal with the smaller grain size. Then, the authors attempted to consider these problems by numerical calculation based on two-, three- and multi-grain-size models shown in Fig. $2^{12-16}$. This paper reviews these results by using a few new figures as well as the main figures in the previous reports ${ }^{12-16}$.

\section{Derivations of Grain Growth Equations in Two-, Three- and Multi-Grain-Size Models}

2.1 Preconditions on the mechanisms of grain growth and rate-determining step

The mechanism of the WC grain growth of hardmetal is accepted in general to be Ostwald ripening ${ }^{7-9)}$. Due to an increase in the equilibrium solubility of solid phase into liquid phase with decreasing size or surface curvature radius of solid grain, the following three steps repeat continuously ${ }^{8.97}$, i.e., (I) solution of smaller solid grains into liquid phase, (II) diffusion of the solute in the liquid phase from the smaller grains to the larger grains, and (III) precipitation of the solute onto the larger grains. Then, the smaller grains gradually disappear, the larger grains gradually grow, and thus the mean grain size increases with increasing sintering time.

The increase in the equilibrium solubility of dispersed grains with decreasing grain size has been explained based on thermodynamics usually for spherical-grain ${ }^{8.9)}$. This concept is thought to be also applicable to WC grains of rectangular prism as follows, when the solubility dependence on grain size is understood from the viewpoint of atomistic process. The equilibrium solubility of dispersed grains into the matrix is considered to be determined by the balance between the dissolving and precipitating rates of solid grain atoms. The dissolving rate especially increases with decreasing grain size or surface curvature radius, due to the increase of dangling atomic-bond density
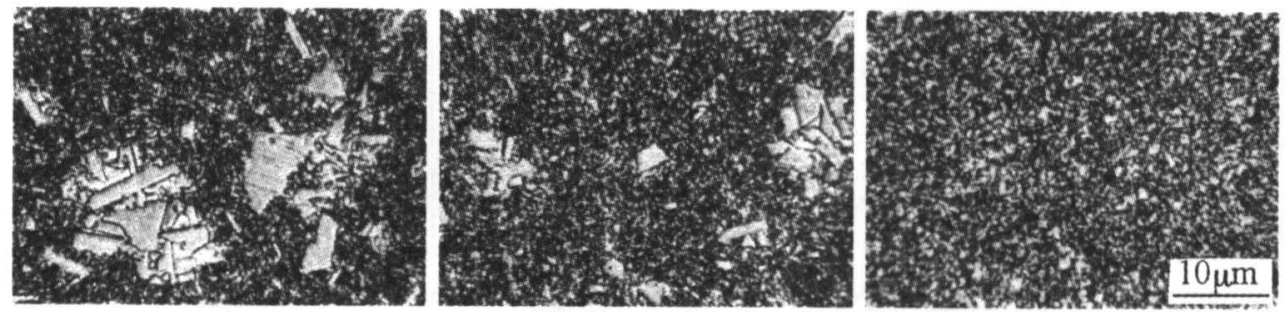

Fig.1 Example of optical micrographs of WC- 0.5 mass $\%-10$ mass $\%$ Co fine grained hardmetals ${ }^{6}$. The WC starting powders with 0.15 and $0.19 \mu \mathrm{m}$ were prepared by severely ball-milling the powder of $0.50 \mu \mathrm{m}$.

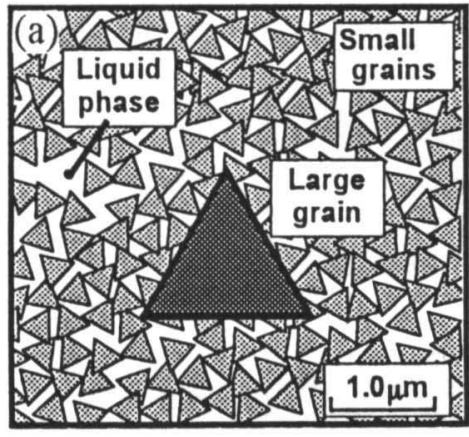

2-grain-size model

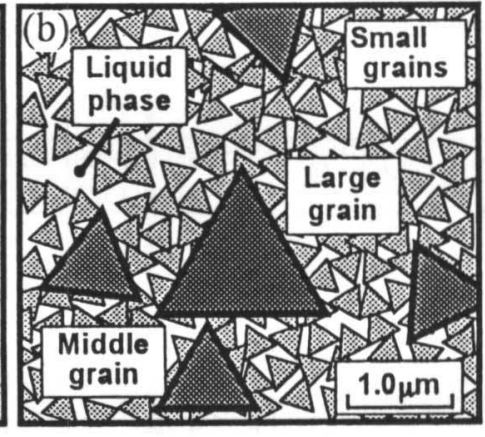

3-grain-size model

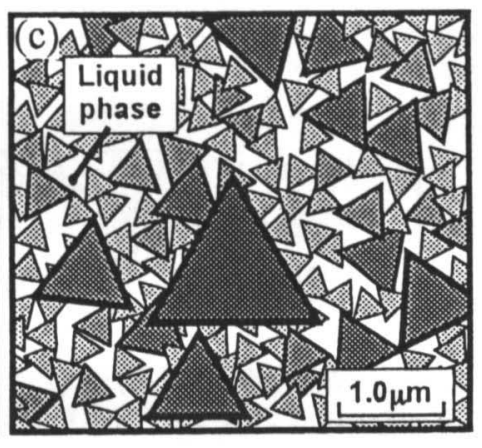

Multi-grain-size model

Fig.2 Alloy models with two-, three- and multi-grain sizes . 
irrespective of the grain shape, as schematically shown in Fig. 3: the atoms in the surface dissolve into the matrix solvent more easily with increasing dangling atomic-bond density. Namely, the dangling atomic-bond density of WC grains increases with decreasing grain size in the same way as that of spherical grain.

As for the rate-determining step of WC grain growth in fine grained WC-Co hardmetal doped with VC is considered to be the interfacial reaction (precipitation), based on the following experimental result and the mechanism for the grain growth inhibition by VC. The experimental result showed that a linear relation was observed between $\bar{d}_{t}^{2}$ and $t$ in the grain size range of $0.21 \sim 0.55 \mu \mathrm{m}$, as shown in Fig. ${ }^{6)}$. The mechanism for the grain growth inhibition is considered as follows. The precipitation of WC solute onto larger WC grains is not possible, unless the $\mathrm{V}$ atoms absorbing on the surface of WC grains desorp, as schematically shown in Fig. $5^{17}$, because the solubility of VC into WC lattice is negligibly small ${ }^{18}$. It is needless to say that the concentration of the solute except near the interface of the larger grains is always the equilibrium solubility of the smallest grains in the alloy system where the rate-determining step of Ostwald ripening is the

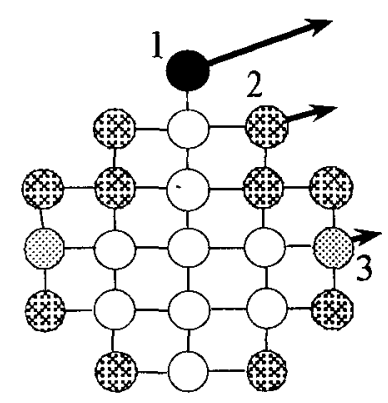

Sphere

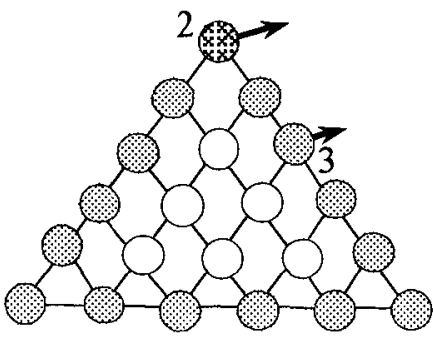

Rectangular prism
Fig.3 Schematic atomistic explanation for the increase of equilibrium solubility accompanied by decreasing grain size for rectangular prism grain as well as spherical grain. The dangling atomic-bond density increases with decreasing gain size or surface curvature radius for rectangular WC grain as well as shperical grain. The easiness of solution or debonding is expressed with the length of arrows or numerals. interface reaction (precipitation), and that the concentration difference for the driving force of precipitation onto the larger grains is the solubility difference between the smallest grain and the larger grains ${ }^{12,13)}$.

2.2 Grain growth equations based on two-grain-size model $^{12,13)}$

This model satisfies the following conditions. As shown in the above Fig. 2(a), a great amount of uniform small WC grains and an extremely small amount of uniform large WC grains are dispersed in Co liquid phase: the number of kinds of grain size is two. Here, the large grain is defined as the grain having more than the size twice of the small grain $\left(d_{\mathrm{L}, 0} d d_{\mathrm{s}, 0} \geqq 2\right)$, and "an extremely small amount" means such amount that the mean grain sizes in both the powder and sintered compacts are determined only by the size of small grains and not affected by the size of large grains, even after the grain growth as well as before sintering. This means the concentration of the WC solute in the Co liquid phase is always the equilibrium solubility of small grains, and that the grain size of small grains hardly vary by the sintering because of the extremely small amount of large grains, i.e., the extremely small amount of precipitation onto

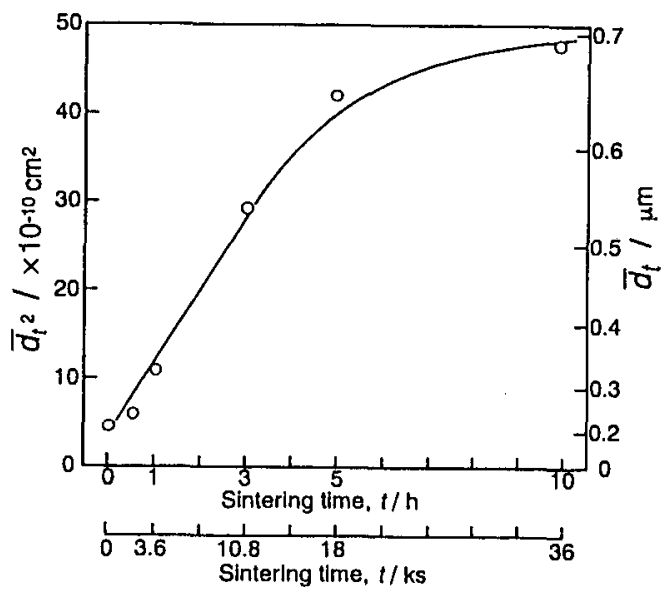

Fig.4 Experimental relation between $\bar{d}_{t}^{2}$ and $t$ in WC-0.5mass\% VC-10mass\%Co alloy (VC content in Co is 5 mass \%) sintered at $1673 \mathrm{~K}$. The mean grain size of WC starting powder was $0.15 \mu \mathrm{m}$. The grain growth from $0.15 \mu \mathrm{m}$ to $0.21 \mu \mathrm{m}$ occurred during rising temperature to the sintering temperature.

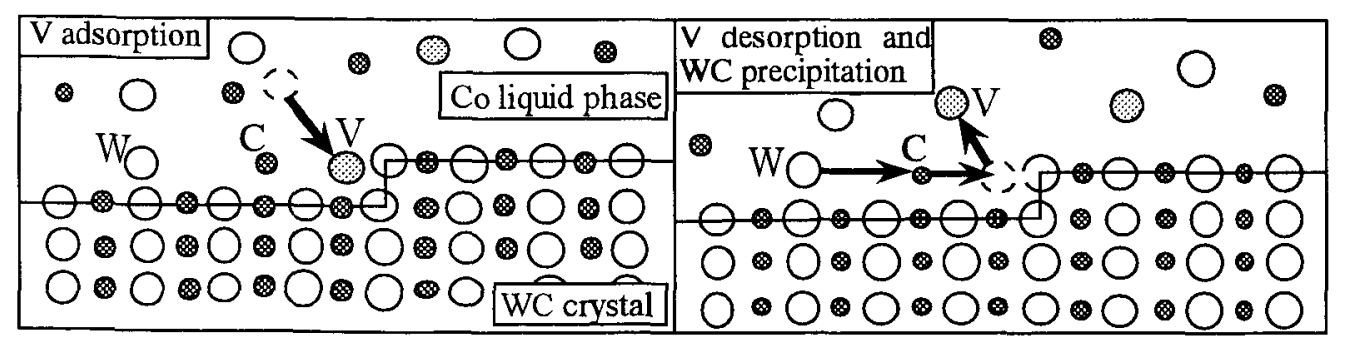

Fig.5 Schematic atomistic explanation for the WC grain growth inhibition by VC. W and C atoms can not move to the stable site (kink), unless $\mathrm{V}$ atom insoluble in WC lattice desorps from the kink or surface. 
large grains or the extremely small amount of dissolution from small grains.

The importance of this alloy model is as follows. The WC powder with an uniform grain size is an ideal one as the starting powder for the fabrication of fine grained hardmetal, because Ostwald ripening basically never occurs in the alloy with uniform grain size. However, a small amount of larger WC grains are thought to mingle into the powder in the actual fabrication factory of hardmetals, where WC starting powders with various sizes are used. Therefore, the numerical calculation based on this alloy model is able to estimate the growth behavior of such mingled larger grains.

The equation which was derived based on the above assumptions or preconditions was the following differential equation $^{12,13)}$.

$$
d_{\mathrm{L} . t+\Delta t}=d_{\mathrm{L}, t}+4\left(2 \sigma \kappa C_{0} V_{m}^{2} / \mathrm{VR} T\right)\left(1 / d_{\mathrm{S} .0}-1 / d_{\mathrm{L}, t}\right) \Delta t
$$

The values of $\sigma, \kappa$ and $v$ in the first parentheses of the coefficient of $\Delta t$ in the right side of this equation are not known for WC-Co hardmetals doped with VC and thus equation (2) is not able to calculate the value of $d_{\mathrm{L}, t}$. However, when the coefficient of the first parentheses of $\Delta t$ in equation (2) is compared with the coefficient of $t$ in equation (1), the former is known to be equal to $81 / 32$ times of the latter. Therefore, equation (2) is rewritten as the following by using the coefficient in equation (1), i.e., $K$ in equation ( $\left.1^{\prime}\right)$. This finding is one of the keypoints in this study, together with the derivations of the above equation and various equations shown hereafter.

$$
d_{\mathrm{L}, t+\Delta t}=d_{\mathrm{L}, t}+(81 / 32) K\left(1 / d_{\mathrm{S}, 0}-1 / d_{\mathrm{L}, t}\right) \Delta t
$$

These equations (2) and (2') are of course applicable to general grain dispersed alloys. The concrete value of $K$ for WC-10mass\%Co hardmetal doped with 0.5 mass $\%$ of VC can be estimated from a linear part of $\bar{d}_{t}^{2}-t$ correlation line in our previous report ${ }^{6}$ : the linear part was obtained in WC grain size range of $0.21 \sim 0.55 \mu \mathrm{m}$ or in the sintering time range of $0 \sim 14.4 \mathrm{ks}$ when the grain growth inhibitor is considered to be uniformly dissolved in the Co liquid phase ${ }^{12,13)}$. The 0.5 mass\% ( 5 mass\% in Co phase) is the content where the effect of the grain growth inhibition of VC becomes nearly saturated and also an appropriate one from the view point of the fracture strength of the alloy. The estimated value of $K$ is $2.18 \times 10^{-2} \mu \mathrm{m}^{2} / \mathrm{ks}\left(2.18 \times 10^{-14}\right.$ $\mathrm{m}^{2} / \mathrm{ks}$ ). Then, equation $\left(2^{\prime}\right)$ can be rewritten by substituting $K$ for $2.18 \times 10^{-2}$ as follows:

$$
d_{\mathrm{L}, t+\Delta t}=d_{\mathrm{L}, t}+5.52 \times 10^{-2}\left(1 / d_{\mathrm{S}, 0}-1 / d_{\mathrm{L}, t}\right) \Delta t
$$

where the units of grain size and time are " $\mu \mathrm{m}$ " and "ks", respectively. It is clear that the numerical calculation of the growth of the large grains $\left(d_{\mathrm{L}, t+\Delta t}\right)$ or abnormal grain growth in the hardmetal with a kind of bimodal grain-size distribution is possible by using equation (2") as a function of $d_{\mathrm{S} .0}$ and $d_{\mathrm{L}, 0}$.

Additionally describing, the number of equations for deriving equation (2) is 7 .

2.3 Grain growth equations based on three-grain-size model $^{14,15)}$

This model is one where middle grains are added to the above two-grain size alloy model, as shown in Fig. 2(b). The equations derived for the growth of large and middle grains before and after the disappearance of the small grain, the extinction of small grain and the mean grain size are the following.

The equations before the disappearance of small grains are as follows:

$$
\begin{aligned}
& d_{\mathrm{M}, t+\Delta t}= d_{\mathrm{M}, t}+5.52 \times 10^{-2}\left(1 / d_{\mathrm{S} .0}-1 / d_{\mathrm{M}, t}\right) \Delta t \\
& d_{\mathrm{L}, t+\Delta t}= d_{\mathrm{L}, t}+5.52 \times 10^{-2}\left(1 / d_{\mathrm{S}, 0}-1 / d_{\mathrm{L}, t}\right) \Delta t \\
& d_{\mathrm{S}, t+\Delta t}=d_{\mathrm{S} . t}-5.52 \times 10^{-2}\left\{d_{\mathrm{M}, t}^{2} N_{\mathrm{M}, t} \cdot\left(1 / d_{\mathrm{S} .0}-1 / d_{\mathrm{M}, t}\right)\right. \\
&\left.\quad+d_{\mathrm{L}, t}^{2} N_{\mathrm{L}, t} \cdot\left(1 / d_{\mathrm{S}, 0}-1 / d_{\mathrm{L}, t}\right)\right\} \cdot \Delta t /\left(d_{\mathrm{S}, t}^{2} N_{\mathrm{S}, t}\right) \\
& \bar{d}_{t}=d_{\mathrm{S}, t} N_{\mathrm{S}, t}+d_{\mathrm{M}, t} N_{\mathrm{M}, t}+d_{\mathrm{L}, t} N_{\mathrm{L}, t}
\end{aligned}
$$

where $N_{S, r}, N_{\mathrm{M}, r}$ and $N_{\mathrm{L}, r}$ are the number fractions of small, medium and large grains, respectively.

The equations after the disappearance of small grains are as follows.

$$
\begin{aligned}
& d_{\mathrm{L}, t+\Delta t}=d_{\mathrm{L}, t}+5.52 \times 10^{-2}\left(1 / d_{\mathrm{M}, 0}-1 / d_{\mathrm{L}, t}\right) \Delta t \\
& d_{\mathrm{M}, t+\Delta t}=d_{\mathrm{M}, t}-5.52 \times 10^{-2} \\
& \quad\left\{d_{\mathrm{L}, t}^{2} N_{\mathrm{L}, t} \cdot\left(1 / d_{\mathrm{M}, 0}-1 / d_{\mathrm{L}, t}\right)\right\} \cdot \Delta t /\left(d_{\mathrm{M}, t}^{2} N_{\mathrm{M}, t}\right) \\
& \bar{d}_{t}=d_{\mathrm{M}, t} N_{\mathrm{M}, t}+d_{\mathrm{L}, t} N_{\mathrm{L}, t}
\end{aligned}
$$

The term of " $5.52 \times 10^{-2 "}$ in these equations and also in the latter equations (10) and (11) is originally of course "(81/ 32) $K "$. Additionally, the total number of equations necessary for deriving these equations were 23 .

2.4 Derivation of grain growth equations based on multigrain-size model $^{16)}$

The multi-gain-size model consists of grains with numerrous sizes as shown in Fig. 2(c). In this model, the variables and notations are defined as follows. The initial number of size is $N_{0}$; the number of descriminating the grain size is " $i$ " ( $\left.i \leqq N_{0}\right)$; the size of grain " $i$ " at the time $t$ is $d_{\mathrm{i},}$; 
the initial total number of grains is $n_{0}$; the total number of grains at time $t$ is $n_{t}$, which decreases with increasing $t$ due to the disapearance of small grains; the total number of grains with the size " $i$ " at time $t$ is $n_{\mathrm{i}, r}$ and the ratio of $n_{\mathrm{i}, r} / n_{r}$ is $F_{\mathrm{i}, t}$, which corresponds to $N_{\mathrm{S}, t}, N_{\mathrm{M}, t}$ and $N_{\mathrm{L}, t}$ in three-grainsize model. Based on the above preconditions that the ratedetermining step of WC grain growth is the interface reaction (precipitation) and that the solubility of WC solute is always the equilibrium solubility of the smallest grains, the growth equation of grains " $i$ " except for the smallest grains, the extinction equation of the smallest grains and the equation of the mean grain size were derived. They are the following, respectively.

$$
\begin{aligned}
& d_{\mathrm{i}, t+\Delta t}=d_{\mathrm{i}, t}+5.52 \times 10^{-2}\left(1 / d_{\min , 0}-1 / d_{\mathrm{i}, t}\right) \Delta t \\
& d_{\text {min }, t+\Delta t}=d_{\text {min }, t}- \\
& \times\left[\sum_{i=\text { min }+1}^{N_{0}}\left\{d_{\mathrm{i}, t}^{2} F_{\mathrm{i}, t}\left(1 / d_{\text {min }, 0}-1 / d_{\mathrm{i}, t}\right)\right\}\right] \Delta t \\
& \bar{d}_{t}=\sum_{\mathrm{i}=\text { min }}^{N_{0}}\left(F_{\mathrm{i}, t} \cdot d_{\mathrm{i}, t}\right)
\end{aligned}
$$

Additionally, the total number of equations necessary for deriving these three equations were 18 .

\section{Results of Numerical Calculation and Consideration}

The computer programs for the calculation of the left term, i.e., $d_{\mathrm{L}, t+\Delta t}, d_{\mathrm{i}, t+\Delta t}, d_{\min , t+\Delta t}, \bar{d}_{t}$, etc., in equations (2") (12) for the above three kinds of models were made (the number of lines are about 120,400 and 500 , respectively), and then installed into a personal computer (NEC PC 9821). Then, those values for WC- 0.5 mass $\%$ VC- 10 mass\% $\%$ hardmetal in the case of usual sintering condition, i.e., at $1673 \mathrm{~K}$ for $3.6 \mathrm{ks}$, were computed.

\subsection{Results based on two-grain-size model ${ }^{12,13)}$}

Fig. 6 shows the calculation result on the effect of $d_{s, 0}$ on $d_{\mathrm{L}, 3.6}$ at various $d_{\mathrm{L}, 0}$. The $d_{\mathrm{L}, 3,6}$ increases naturally with decreasing $d_{\mathrm{S}, 0}$ for each $d_{\mathrm{L}, 0}$. It is noted, however, that $d_{\mathrm{L}, 3.6}$ sharply increases below $0.1 \sim 0.2 \mu \mathrm{m}$ : for example at $d_{\mathrm{s}, 0}=$ $0.035 \mu \mathrm{m}(35 \mathrm{~nm}), d_{\mathrm{L}, 3.6}$ extremely increases up to about $5 \sim$ $6 \mu \mathrm{m}$, regardless of $d_{\mathrm{L}, 0 .}$. Above $0.3 \mu \mathrm{m}, d_{\mathrm{L}, 3.6}$ is in general below $1.0 \mu \mathrm{m}$, when $d_{\mathrm{s}, 0}$ is near $d_{\mathrm{L}, 0}$.

Fig. 7 shows schematic microstructures which were drawn by using the values of $d_{\mathrm{L}, 3.6}$ and $d_{S, 3.6}\left(=d_{5,0}\right)$ shown in Fig. 6. The shape and size of WC grains are of an equilateral trianglular prism having area equal to that of

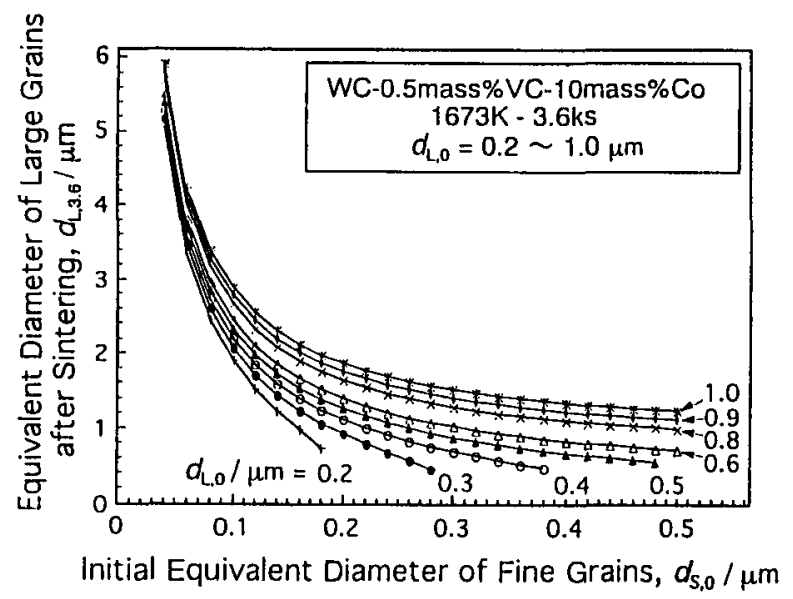

Fig.6 Calculation result of the effect of $d_{\mathrm{S}, 0}$ on $d_{\mathrm{L}, 3.6}$ at various $d_{\mathrm{L}, 0}$ in two-grain size alloy model.

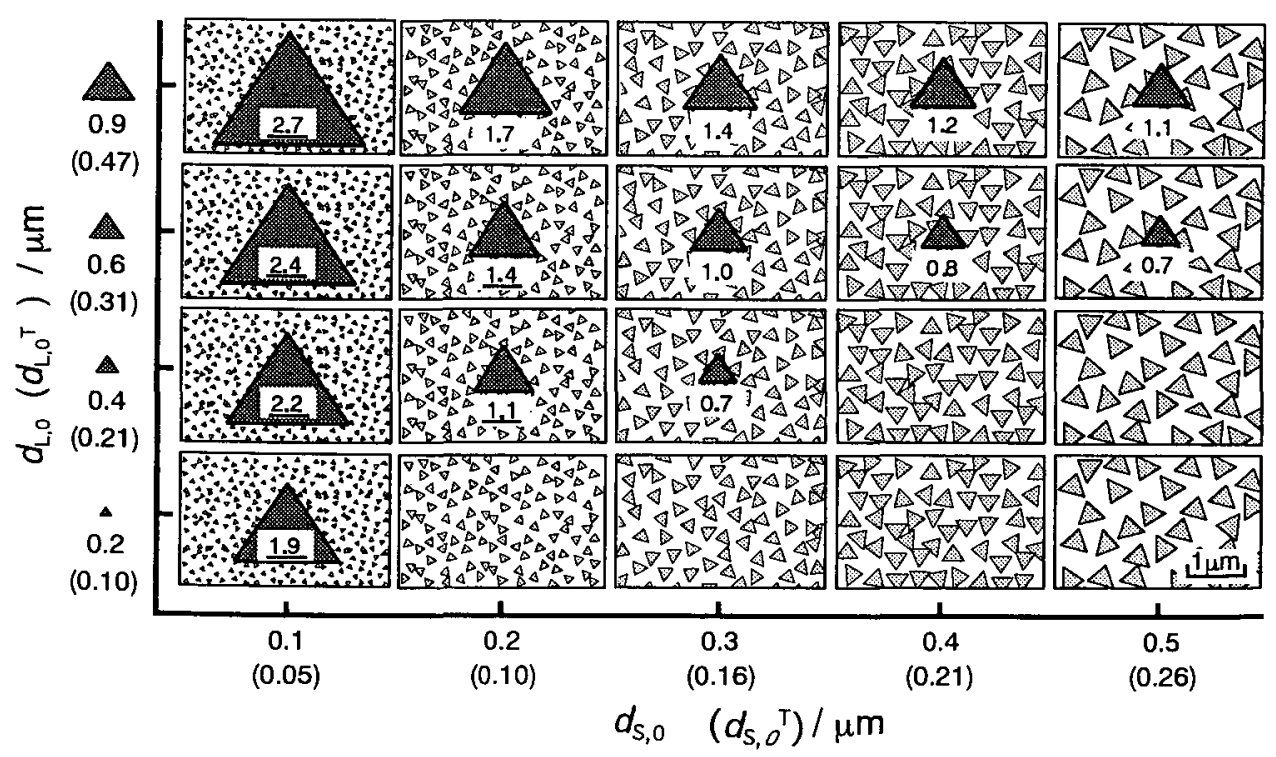

Fig.7 Schematic microstructures which were drawn by using the values of $d_{\mathrm{L}, 3.6}$ and $d_{\mathrm{S}, 0}$ shown in Fig. 6 . The large grains with underlined grain-size-numerals are abnormally-grown grains $\left(d_{\mathrm{L}, 3.6} / d_{\mathrm{L}, 0}\right) \geqq 2$, i.e., $\left.d_{\mathrm{L}, 3,6} / d_{\mathrm{S}, 0} \geqq 4\right)$. 
the cross-sectional plane (circle) of the equivalent spherical grains with the same volume. Concretely, when the diameter of equivalent spherical grain on the observed plane is expressed as $d_{\mathrm{c}}\left(=2 / 3 \times d_{\mathrm{L}, t}\right)$ and the length of the perpendicular from the center of gravity to the base is expressed as $d_{\mathrm{L}, \mathrm{r}}^{\mathrm{T}} / 2$, the relation of $d_{\mathrm{L}, f}^{\mathrm{T}}=0.78 d_{\mathrm{c}}\left(=0.52 d_{\mathrm{L}, t}\right)$ holds ${ }^{12.13}$. In Fig. 7, the microstructures where the large grain looks considerably larger than the small grains seem to correspond to the case of $d_{\mathrm{L}, 3.6}^{\mathrm{T}} / d_{\mathrm{s}, 3.6}^{\mathrm{T}}\left(\doteqdot d_{\mathrm{L}, 3.6} / d_{\mathrm{L}, 0}\right) \geqq 4$. Then, "the abnormal grain growth" was defined in this model as the growth of the large grains satisfying the condition of $d_{\mathrm{L}, 3.6} / d_{\mathrm{L}, 0} \geqq 2$, because $d_{\mathrm{L}, 0}$ was defined to be larger than $2 d_{\mathrm{s}, 0}$, as mentioned above.

From Figs. 6 and 7 and also the relation between $d_{\mathrm{L}, 3.6} / d_{\mathrm{L}, 0}$ and $d_{\mathrm{s}, 0}{ }^{12,13)}$, the following are known: (1) For $d_{\mathrm{s}, 0} \leqq 0.1 \mu \mathrm{m}$, $d_{\mathrm{L}, 3.6} / d_{\mathrm{L}, 0}$ ratio is always larger than 3.0 irrespective of the values of $d_{\mathrm{L}, 0}$; abnormal grain growth always occurs. (2) For $d_{\mathrm{S}, 0}=0.2 \mu \mathrm{m}, d_{\mathrm{L}, 3 . \mathrm{o}} / d_{\mathrm{L}, 0}$ ratio becomes larger than $2.0 \sim$ $2.7\left(d_{\mathrm{L}, 3.6}=1.1 \sim 1.4 \mu \mathrm{m}\right)$ at $d_{\mathrm{L}, 0}=0.4 \sim 0.6 \mu \mathrm{m}$; abnormal grain growth occurs. In the case of $d_{\mathrm{L}, 0}=0.9 \sim 1.0 \mu \mathrm{m}, d_{\mathrm{L}, 3.6} / d_{\mathrm{L}, 0}$ ratio is smaller than $1.9\left(d_{\mathrm{L}, 3.6}=1.7 \sim 1.9 \mu \mathrm{m}\right)$, although $d_{\mathrm{L}, 3.6} / d_{\mathrm{S}, 0}$ ratio is as large as $8.5 \sim 9.5$. (3) For $d_{\mathrm{S}, 0} \geqq 0.3 \mu \mathrm{m}$, $d_{\mathrm{L}, 3.6} / d_{\mathrm{L}, 0}$ ratio is $1.0 \sim 1.7\left(d_{\mathrm{L}, 3.6}=0.45 \sim 1.5 \mu \mathrm{m}\right)$, i.e., less than 2.0, irrespective of the values of $d_{\mathrm{L}, 0}$ : abnormal grain growth does not occur. The large grains observed in the case of $d_{\mathrm{L}, 3.6} / d_{\mathrm{L}, 0}<2$ is not caused by the abnormal grain growth, but by the pre-existence of large grains in the starting powder. These calculation results are in good accordance with the experimental result shown in Fig. 1.

Based on the above results, the hypothesis (ii), i.e., "the non-uniform distributions of grain growth inhibitor", was considered not to be the case for all calculation results, because the value of $K$ used in equation ( $\left.2^{\prime \prime}\right)$ is one obtained for the case where such non-uniform distributions were considered not to exist, as described above. The hypotheses (i) and (iii) were thought to be appropriate to the following results, respectively: (1) The abnormal grain growth in the case of $d_{\mathrm{S}, 0} \leqq 0.1 \mu \mathrm{m}$ and also in the case of $d_{\mathrm{S}, 0}=0.2 \mu \mathrm{m}$ and $d_{\mathrm{L}, 0}=0.4 \sim 0.6 \mu \mathrm{m}$ are substantial as far as 0.5 mass $\%$ VC ( 5 mass\% in Co phase) is used as a grain growth inhibitor, because these occur even when the size of the large grains becomes over the size only twice of the fine grains, and (2) the abnormal grain growths in the case of $d_{\mathrm{s}, 0}=0.2 \mu \mathrm{m}$ and $d_{\mathrm{L}, 0}$ of $0.6 \sim 0.8 \mu \mathrm{m}$ are due to wide grain size distribution, or, large ratio of $d_{\mathrm{L},} / d_{\mathrm{s}, 0}$. The large grains with $d_{\mathrm{L},} d d_{\mathrm{S}, 0} \geqq 4$ in the cases except for the above conditions are not due to the abnormal grain growth, but to the existence of large grains in the WC starting powder: the large grains observed at $\bar{d}_{0}=0.27 \mu \mathrm{m}$ in the previous report ${ }^{6)}$ was thought to correspond to this case. These considerations suggest that the present strategy of selecting the mean grain size of above $0.5 \mu \mathrm{m}$ is appropriate as far as VC is used mainly as grain growth inhibitor, for the stable production of fine grained hardmetals without abnormally grown large grains. The above simulation results also suggest that the production of fine grained hardmetals with grain size of $0.3 \mu \mathrm{m}$ is possible, if the production conditions are severely controlled.

The value of $K$ necessary for inhibiting abnormal grain growth, for example, even in the case of $d_{\mathrm{s}, 0}=0.1 \mu \mathrm{m}$ was estimated to be less than $0.336 \times 10^{-2} \mu \mathrm{m}^{2} / \mathrm{ks}$, which is about $1 / 6$ times of that for 0.5 mass $\%$ ( 5 mass $\%$ in Co phase) VC addition as above. The findings of new grain growth inhibitors or new hard substances having $K$ of such lower value should be necessary, if we wish to fabricate hardmetals with grain size below $0.1 \mu \mathrm{m}$ stably. 3.2 Results based on three-grain-size model ${ }^{14,15)}$

Fig. 8 shows the effect of the amount of middle grains ( $W_{\mathrm{M}, 0}$, weight or volume fraction in $\mathrm{WC}$ ) on the relation between $d_{\mathrm{L}, 3.6}$ and $d_{\mathrm{S}, 0 .}$. The relation at $W_{\mathrm{M}, 0}=0$ of course is the same as that in the above two-grain-size model. The $d_{\mathrm{L}, 3.6}$ becomes almost constant below a critical value of $d_{\mathrm{S}, 0}$ and the critical $d_{\mathrm{S}, 0}$ increases with increasing $W_{\mathrm{M}, 0}$. This is due to the disappearance of small grains during sintering: the disappearance occurs sooner with increasing $W_{\mathrm{M}, 0}$. The disappearance causes the decrease of the grain growth rate of large grains, because the WC solute-concentration difference between middle and large grains is smaller than that between small and large grains.

Fig. 9 shows the schematic microstructure as a function of $d_{\mathrm{S}, 0}$ and $W_{\mathrm{M}, 0}$. It is noted that the microstructure or the mean grain size becomes very coarse in the case of relatively

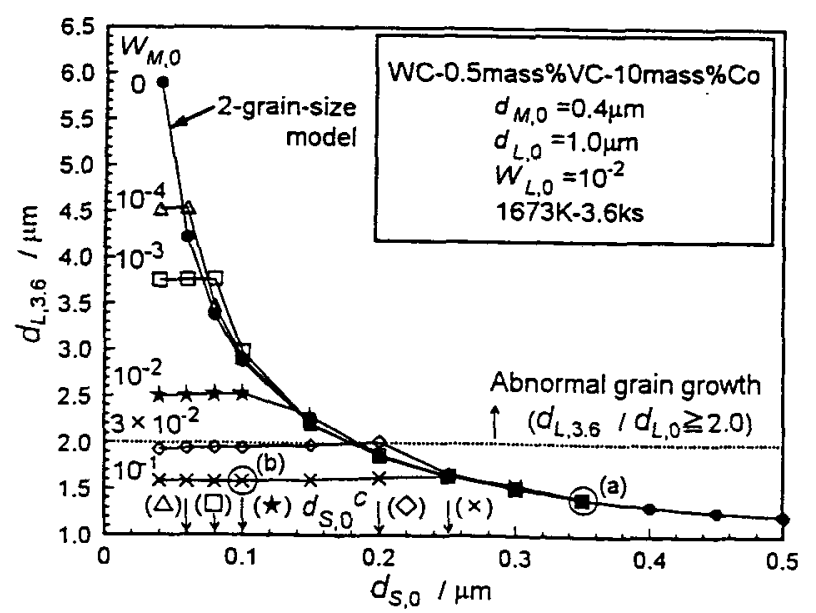

Fig.8 Calculation result on effect of the middle grain amount $\left(W_{\mathrm{M} .0}\right)$ on $d_{\mathrm{L}, 3,6}-d_{\mathrm{S}, 0}$ relation obtained in two-grain size alloy model. 
large amount of $W_{\mathrm{M}, 0}$, although the microstructure in the case of small $W_{\mathrm{M}, 0}$ is nearly the same as that in the above two grain size alloy model. This is of course due to the disappearance of small grains as above mentioned.

\subsection{Results based on multi-grain-size model ${ }^{16)}$}

The following two kinds of density functions were used as the initial grain size distribution: (1) monomodal (normal) distribution, which is generally observed in commercial WC powders, and (2) bimodal distribution consisting of two kinds of normal distributions, which seemed to cause the abnormal grain growth based on the result of the above two-grain-size model. Their distributions, i.e., the relation between the grain size diameter $\left(d_{i, 0}\right)$ and the frequency $\left(F_{\mathrm{i}, 0} \equiv n_{\mathrm{i}, 0} / n_{0}\right)$ which is included in the above equation (11) are expressed at $t=0$ by the following equations, respectively:

$$
\begin{aligned}
F_{\mathrm{i}, 0} & =\left\{1 /\left(\sqrt{2 \pi} \sigma_{0}\right)\right\} \exp \left\{-\left(d_{\mathrm{i}, 0}-\bar{d}_{0}\right)^{2} /\left(2 \sigma_{0}^{2}\right)\right\} \\
F_{\mathrm{i}, 0} & =\left[\left\{1 /\left(\sqrt{2 \pi} \sigma_{0}^{1 m}\right)\right\} \exp \left\{-\left(d_{\mathrm{i}, 0}-\bar{d}_{0}^{1 m}\right)^{2} / 2\left(\sigma_{0}^{1 m}\right)^{2}\right\}\right. \\
& \left.+\left\{1 /\left(\sqrt{2 \pi} \sigma_{0}^{2 m}\right)\right\} \exp \left\{-\left(d_{\mathrm{i}, 0}-\bar{d}_{0}^{2 m}\right)^{2} / 2\left(\sigma_{0}^{2 m}\right)^{2}\right\}\right] / 2
\end{aligned}
$$

where $\sigma_{0}, \sigma_{0}^{1 m}$ and $\sigma_{0}^{2 m}$ are the standard deviations for monomodal distribution and the first- and second-modes of bimodal distribution respectively, $d_{\mathrm{i}, t}$ is the size of the grain " $i$ ", and $\bar{d}_{0}, \bar{d}_{0}^{1 m}$ and $\bar{d}_{0}^{2 m}$ are the initial mean grain sizes. Fig. 10 shows an example of $F_{i, 0}$, i.e., the initial grain size distribution curve, in a monomodal distribution for $\bar{d}_{0}=$ $0.2 \mu \mathrm{m}$ as a function of $\sigma_{0}$.

\subsubsection{Monomodal (normal) distribution}

The calculation was carried out for $\bar{d}_{0}=0.035 \mu \mathrm{m}(35 \mathrm{~nm})$ $\sim 0.5 \mu \mathrm{m}$ and $\sigma_{0}=0.001 \sim 0.075$. Fig. 11 (a) (b) shows the calculation result on the effect of $t$ on the grain size distribution curve at $\sigma_{0}=0.005$ and 0.075 in the case of $\bar{d}_{0}$ $=0.2 \mu \mathrm{m}$. From these figures, etc., the following were clarified: (1)the smaller grains disappear one after another with increasing $t,(2)$ the time for the disappearance of the maximum point in $F_{\mathrm{i}, 0}$ curve becomes shorter with increasing $\sigma_{0}$, and (3) the movement rate of the curve to the larger grain sizes becomes faster with increasing $\sigma_{0}$. The cause for the results (2) and (3) are both the following: with increasing $\sigma_{0},(1)$ the values of $F_{\mathrm{i}, \mathrm{f}}$ for the smaller and larger grains increase, (2) thus, the value of $\left(1 / d_{\min , 0}-1 / d_{\mathrm{i}, t}\right)$ in equations (10) and (11), in other words, the solubility difference between the smaller and larger grains, increases, and (3) then, extinction rate of smaller grains and the growth rate of larger grains both increase.

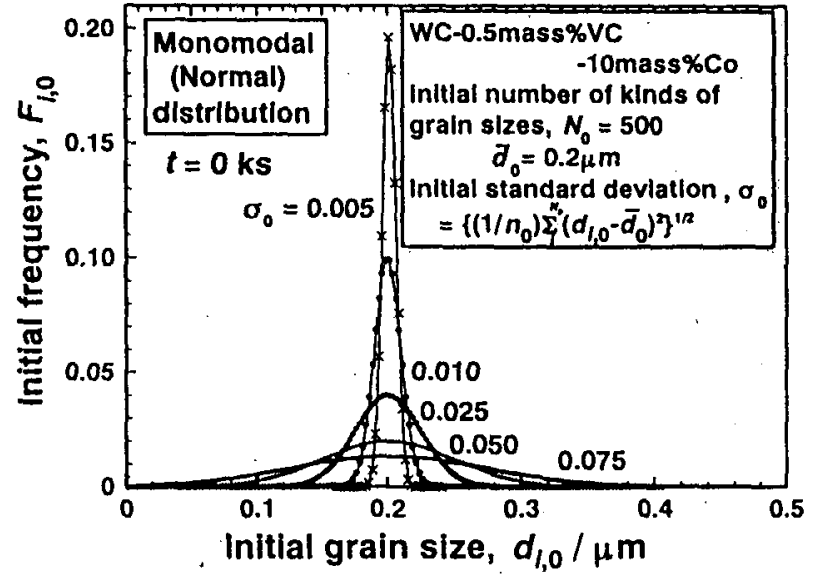

Fig.10 Example of $F_{i, 0}$ curve in a monomodal distribution for $\bar{d}_{0}=$ $0.2 \mu \mathrm{m}$ at several $\sigma_{0}$.

$(4.2,0.34 \mu \mathrm{m})$

$d_{s, 0} / \mu m$
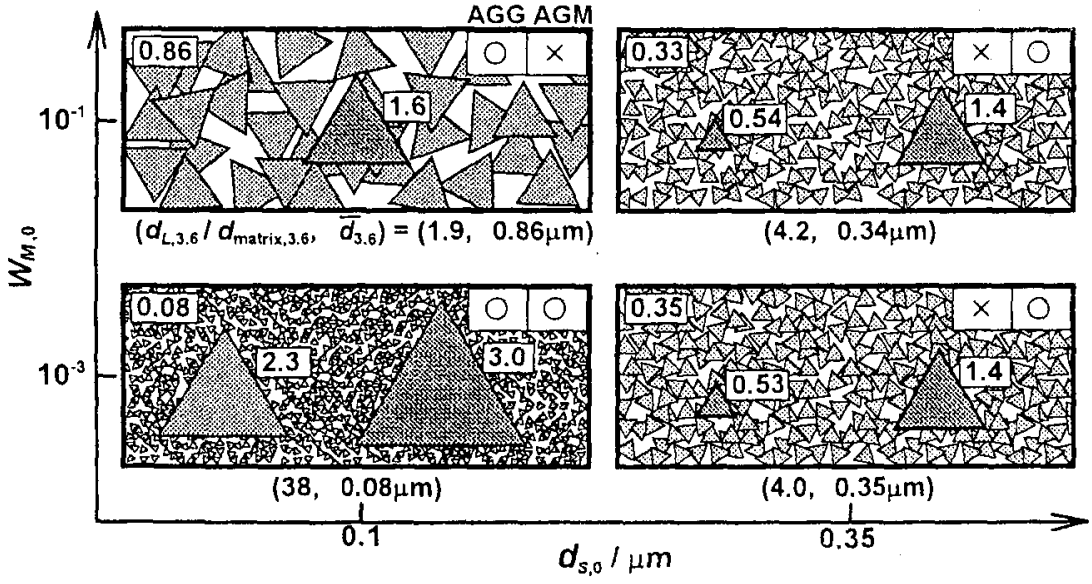

AGG AGM

Fig.9 Schematic microstructure showing effect of $d_{\mathrm{S} .0}$ and $W_{\mathrm{M} .0}$ in three-grain size alloy model. "AGG" and "AGM" means the abnormal grain growth of large grain $\left(d_{\mathrm{L}, 3.0} / d_{\mathrm{L}, 0} \geqq 2\right)$ and abnormal grain microstructure $\left(d_{\mathrm{L}, 3.6} / d_{\mathrm{S}, 0} \geq 4\right)$, respectively. "O" and "X" mean the occurrence or not of such microstructures, respectively. 
Fig. 12 shows the effects of $\sigma_{0}$ and $\bar{d}_{0}$ on the time dependence of $\bar{d}_{t}$. The increment of $\bar{d}_{t}$ for each $\bar{d}_{0}$ increases with increasing $\sigma_{0}$, and $\bar{d}_{\text {, for smaller }} \bar{d}_{0}$ becomes larger than that for larger $\bar{d}_{0}$ at long $t$, when $\sigma_{0}$ for smaller $\bar{d}_{0}$ is larger than that for larger $\bar{d}_{0}$. Mainly owing to such effects, there is a case where $\bar{d}_{3.6}$ shows a minimum at $\bar{d}_{0}$ of $0.2 \mu \mathrm{m}$ in the relation between $\bar{d}_{3.6}$ and $\bar{d}_{0}$, as shown in Fig. 13. The figure also suggests that the $\bar{d}_{3.6}$ becomes about $0.4 \mu \mathrm{m}$, even when the WC starting powder with a mean grain size below $0.1 \mu \mathrm{m}$,

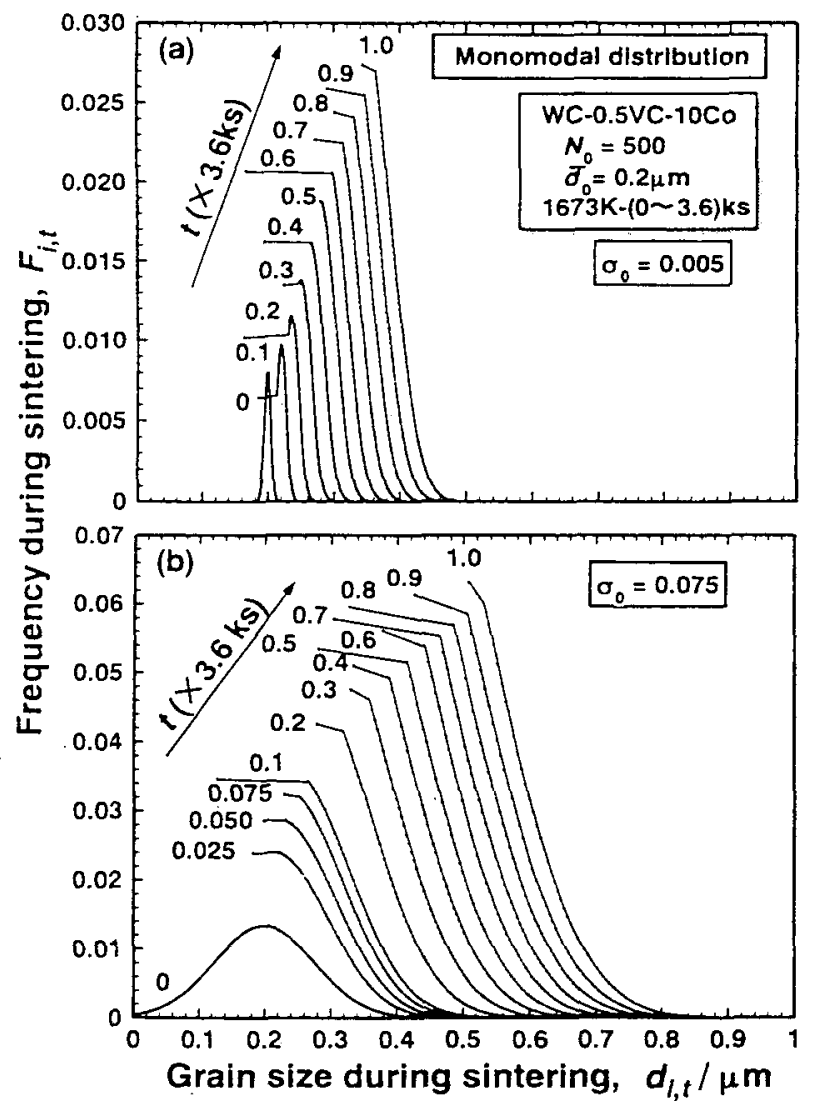

Fig. 11 Calculation result on effect of $\sigma_{0}$ on relation between grain size distribution curve and $t$ in the case of $\bar{d}_{0}=0.2 \mu \mathrm{m}$.

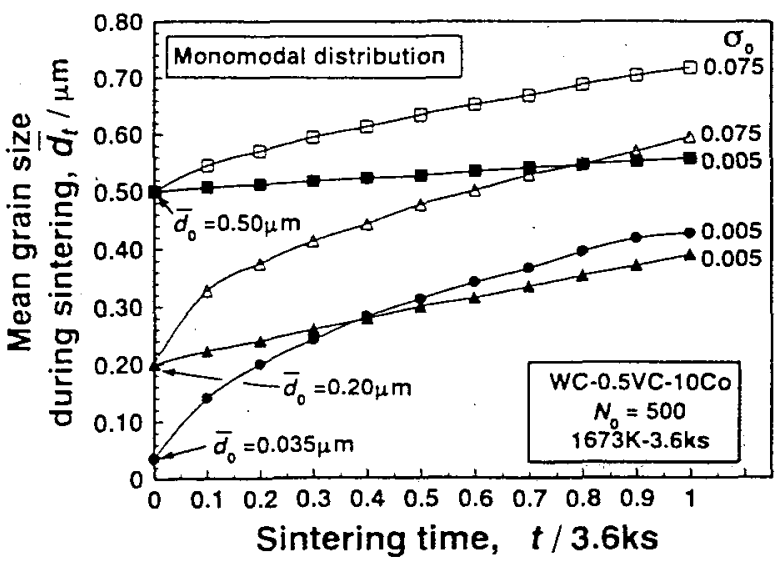

Fig.12 Calculation result on effects of $t$ and $\sigma_{0}$ on $\bar{d}_{t}$. e.g., $0.035 \mu \mathrm{m}(35 \mathrm{~nm})$ is used. This is in close accordance with the following experimental result: the mean grain size of WC-Co hardmetal with VC of 5 mass\% in Co, which was prepared from WC powder with mean grain size of $0.035 \mu \mathrm{m}$ under the sintering condition of $1673 \mathrm{~K}-3.6 \mathrm{ks}$, was about $0.4 \mu \mathrm{m}^{19,20)}$.

These results were similar to those obtained by threegrain-size model.

It was also noted in Fig. 12 that the increment of $\bar{d}_{t}$ with $t$ increases with increasing $\sigma_{0}$. This means that the increment of $\bar{d}_{t}^{2}$ with $t$, i.e., the $K$ value in equation of $\bar{d}_{t}^{2}$ $-\bar{d}_{0}^{2}=K t$ increases with increasing $\sigma_{0}$. This is reasonable, when we consider that the $K$ value becomes zero at $\sigma_{0}=0$ (completely uniform grain size). Furthermore, this can be seen in the experimental result shown in Fig. 4 where the slope, i.e., the $K$ value of $2.18 \times 10^{-2} \mu \mathrm{m}^{2} / \mathrm{ks}$ in the range of $0.21 \sim 0.55 \mu \mathrm{m}$ changed to about $0.3 \times 10^{-2} \mu \mathrm{m}^{2} / \mathrm{ks}$ above $0.55 \mu \mathrm{m}$. The initial $\sigma_{0}$ value of the alloy ${ }^{6)}$ used in this study could not be measured, because the alloy specimens had already been abondaned about eighteen years ago. However, the value was suggested to be $0.001 \sim 0.01$, based on the relations of $\bar{d}_{t}-t$ and $\bar{d}_{3.6}-\bar{d}_{0}$ calculated by using equation of $d_{t}^{2}-d_{0}^{2}=K t$ ( $K$ is $2.18 \times 10^{-2} \mu \mathrm{m} / \mathrm{ks}$ ). Namely, the relations both coincided well with those by mult-grainsize model at $\sigma_{0}$ value of $0.001 \sim 0.01$. It should be necessary to study the dependence of $K$ value on $\sigma_{0}$ from experimental viewpoint.

\subsubsection{Bimodal distribution}

Fig. 14 shows the effect of $d_{0}^{2 m}(0.20$ and $0.60 \mu \mathrm{m})$ on the relation between bimodal grain size distribution curves and $t$ for the initial conditions of $\bar{d}_{0} \doteqdot d_{0}^{1 m}=0.1 \mu \mathrm{m}, \sigma_{0}^{1 m}=$ $\sigma_{0}^{2 m}=0.005$ and $F_{0}^{1 m} / F_{0}^{2 m}=1 / 10^{-7}$, i.e., $\log \left(F_{0}^{1 m} / F_{0}^{2 m}\right)=7$. In the figure, the second distribution curve is denoted by a circle showing its location, because the height is negligibly small. The dependence of the shape and location of the

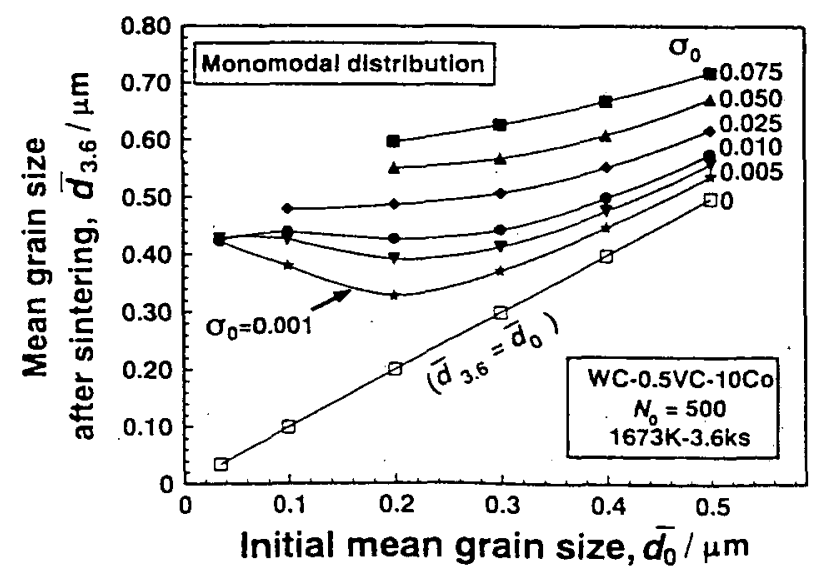

Fig.13 Calculation result on effects of $\bar{d}_{0}$ and $\sigma_{0}$ on $\bar{d}_{3.6}$. 
first distribution curve on $t$ was hardly affected by $d_{0}^{2}$ and was nearly the same as that in the case of the above monomodal distribution. These are due to the fact that the total growth volume in the second distribution or the total extinction volume in the first distribution is extremely small due to the extreme largeness of $F_{0}^{1 m} / F_{0}^{2 m}$.

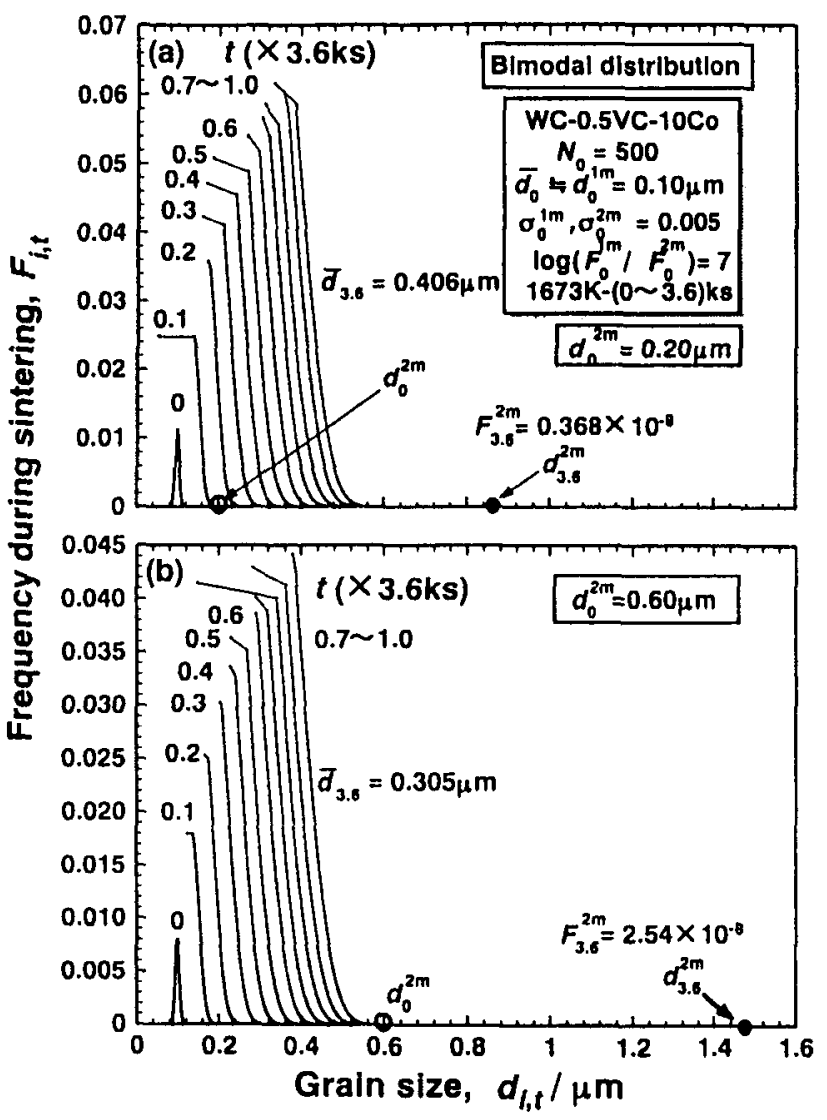

Fig. 14 Calculation result on effect of $d_{0}^{2 m}(0.20$ and $0.60 \mu \mathrm{m})$ on relation between bimodal grain size distribution curves and $t$ for initial conditions of $\bar{d}_{0} \doteqdot d_{0}^{1 m}=0.1 \mu \mathrm{m}, \sigma_{0}^{1 m}=\sigma_{0}^{2 m}=0.005$ and $F_{0}^{1 m} / F_{0}^{2 m}=1 / 10^{-7}$, i.e., $\log \left(F_{0}^{1 m} / F_{0}^{2 m}\right)=7$.

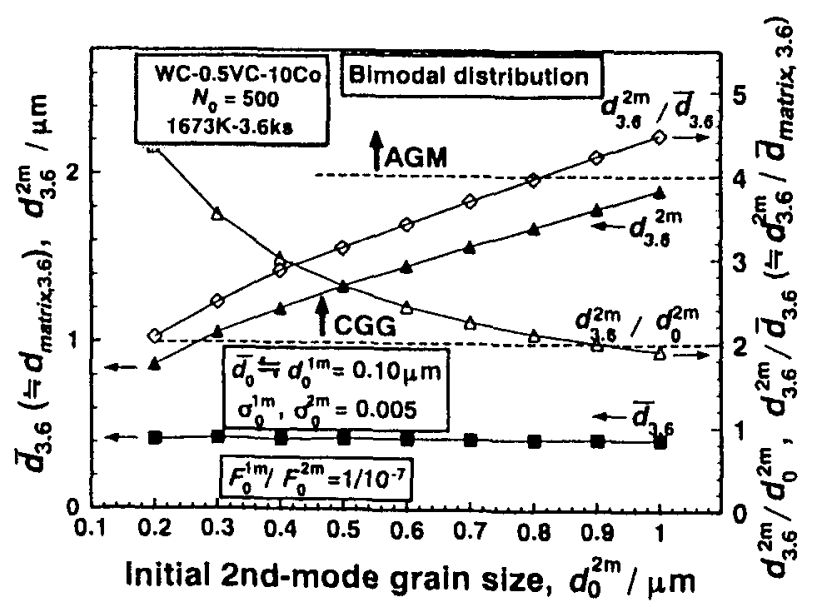

Fig.15 Calcultaion result on effect of $d_{0}^{2 m}\left(=\bar{d}_{0}^{2 m}\right)$ on $\bar{d}_{3.6}\left(\fallingdotseq d_{3.6}^{\mathrm{im}}\right.$

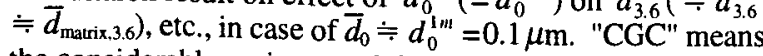
the considerable grain growth in the second mode.
Fig. 15 shows the effect of $d_{0}^{2 m}\left(=\bar{d}_{0}^{2 m}\right)$ on $\bar{d}_{36}\left(\doteqdot d_{36}^{1 m} \doteqdot\right.$ $\left.\bar{d}_{\text {matrix } 3.6}\right), d_{3.6}^{2 m} / \bar{d}_{3.6}$, etc., in the case of $\bar{d}_{0} \doteqdot d_{0}^{1 m}=0.1 \mu \mathrm{m}$. The abnormal grain microstructure (AGM), which is defined as the structure satisfying the condition of $d_{3.6}^{2 m} / \bar{d}_{\text {marrix } 3.6}\left(\fallingdotseq d_{3.6}^{2 m} / \bar{d}_{3.6}\right) \geqq 4$, occurs, when the $d_{0}^{2 m}$ becomes above 0.8 , i.e., $d_{0}^{2 m} / d_{0}^{1 m}$ becomes above 8 . The value is considerably larger than that in the above twograin size model. This can be understood by considering that the grain size in the first mode extremely increases during sintering, leading to the decreasing the solubility difference between smaller grains and larger grains. It was also suggested that the abnormal grain microstructure appears, when $\log \left(F_{0}^{1 m} / F_{0}^{2 m}\right)$ is larger than 4 in the case of $d_{0}^{1 m}=0.1 \mu \mathrm{m}$ and $d_{0}^{2 m}=1.0 \mu \mathrm{m}$.

These results were qualitatively similar to those obtained by two-grain-size model.

\section{Summary}

(1) Numerical calculation equations for the interfacial controlled grain growth, grain extinction and mean grain size on two-, three- and multi-grain-size models, which are applicable to general grain dispersed alloys, were newly derived. Most of these original equations contain unknown parameters such as the interfacial energy, interfacial reaction rate constant and a correction factor which depends on the deviation of the composition from the stoichiometric value, shape and contiguity of solid grains at sintering temperature. However, these can be substituted together by measurable $K$ in a well-known equation of $\bar{d}_{t}^{2}-\bar{d}_{0}^{2}=K t$. Thus, these equations enable the numerical calculation, the results of which suggest valuable answers to the actual problems.

(2) The calculation results showed the following: (i) the abnormal grain growth tends to occur in the case where the initial mean grain size of WC starting powder is below $0.1 \mu \mathrm{m}$ and also the grain size distribution is bimodal with a large grain size difference between two kinds of mode, and (ii) the mean grain size of the alloy becomes so large as about $0.4 \mu \mathrm{m}$, even when the starting WC grain size is $0.035 \mu \mathrm{m}(35 \mathrm{~nm})$. These calculation results are in good accordance with the experimental results.

(3) The above calculation results suggest the following: (i) the present strategy that the mean grain size of fine grained hardmetal is selected to be above $0.5 \mu \mathrm{m}$ seems to be rational as far as $\mathrm{VC}$ is mainly used as grain growth inhibitor from the viewpoint of its stable production. However, the production of hardmetal with grain size of $0.3 \mu \mathrm{m}$ is possible, if the production conditions are 
severely controlled, (ii) the development of WC starting powder with a very narrow grain size distribution is not effective for the mean grain size below $0.1 \mu \mathrm{m}$, and (iii) the finding of new grain growth inhibitor extremely stronger than the present $\mathrm{VC}$ or the syntheses of new $\mathrm{W}$ base hard compound, e.g., W(C,N) are necessary for the development of fine grained hardmetal with grain size below $0.1 \mu \mathrm{m}$.

(4) The ground for the applicability of the Ostwald ripening mechanism to WC grains with triangular prism, the mechanism for the grain growth inhibition of WC by the adition of VC, etc., were also described.

\section{Acknowledgments}

The authors are greatly indebted to Dr. Yoshihiko Doi in Allied Material Corp. in valuable discussion.

\section{References}

1) G.E.Spriggs: "A History of Fine Grained Hardmetal", Int. J. of Refractory Met. and Hard Mat., 13(1995)241255.

2) M.Ueki: Private communication, (2000).

3) K.Hayashi, Y.Fuke and H.Suzuki: "Effects of Addition Carbides on Grain Size...", J. Japan Inst. Powder and Powder Metallurgy, 19(1972)67-71.

4) Product Specification of Tokyo Tungsten Co. Ltd., "WC Fine Powder", (1996).

5) Product Specification of Nanodyne Incorporated, "Mycrocarb Tungsten Carbide Cobalt", New Brunswich, NJ, USA, (1997).

6) H.Suzuki, Y.Fuke and K.Hayashi: "Grain Size of WC in Sintered WC-VC-Co...", J. Japan Inst. Powder and Powder Metallurgy, 19(1972)106-112.

7) R.L.Fullman: Trans. AIME, 197(1953)447-452.

8) C.Wagner: "Theorie der Alterung von Niederschlägen durch Umlösen", Z. Electrochem., 65(1961)S.581-591.
9) R.M.German: Liquid Phase Sintering, Plenum Press, New York, (1985)133-155.

10) C.A.Johnson: "Generalization of Gibbs-Thomson Equation", Surf. Scie., 3(1965)429-444.

11) A.J.Ardell: "Effect of Volume Fraction on Particle Coarsening: Theoretical Considerations", Acta. Met., 20(1972)61-71.

12) N.Matsuoka and K.Hayashi: "A Consideration on Abnormal Grain Growth in Fine Grained WC-Co Hardmetal by Numerical Calculation-Based on Alloy Model with Two kinds of Grain Sizes-", Proceedings of 14th International Plansee Seminar '97 vol. 2, G.Kneringer, et al., Reutte, Austria, Plansee AG., RWF, Werbegesellschaft m.b.H., (1997)609-621.

13) N.Matsuoka and K.Hayashi: "A Consideration ‥on Two Grain Size Alloy Model", J. Japan Inst. Metals, 62(1998)383-389.

14) N.Matsuoka, Y.Doi and K.Hayashi: "A Consideration on Grain Growth ‥with Three Kinds of Grain Sizes", J. Japan Inst. Powder and Powder Metallurgy, 45(1998) 535-543.

15) N.Matsuoka and K.Hayashi: "Processing and Fabrication of Advanced Materials VI", Vol. 1, Edited by K.A.Khor, et.al., IOM Comunuications Ltd., London, (1998)35-47.

16) N.Matsuoka, Y.Doi and K.Hayashi: "A Consideration on Grain Growth $\cdots$ on Multi Grain' Size Model", J. Japan Inst. Powder and Powder Metallurgy, 45(1998)544552.

17) K.Hayashi: "Handbook for Ultrafine Particles", FujiTechno-System, Tokyo, (1990)509-515.

18) H.Suzuki and K.Hayashi: Hardmetals and Sintered Hard Materials -Fundamentals and Applications-, Edited by H.Suzuki, Maruzen Co., Tokyo, (1986)86.

19) N.Asada: Private communications, (1998).

20) Y.Kubo: Private communications, (2000). 